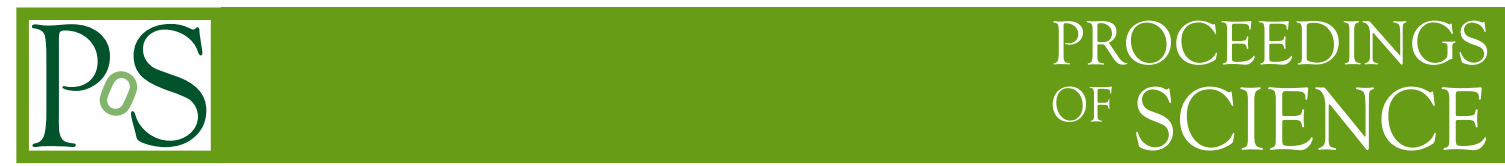

\title{
Higgs and New Physics at ATLAS and CMS
}

\section{Stefano Giagu*}

Sapienza Università di Roma and INFN Roma, Roma-IT

E-mail: stefano.giagudcern.ch

\begin{abstract}
Results of the search for new physics effects in proton-proton collision data at $\sqrt{s}=13 \mathrm{TeV}$ collected by the ATLAS and CMS detectors at the Large Hadron Collider are presented. Measurement of the Standard Model Higgs boson in different production and decay channels, as well as direct Beyond-Standard-Model searches in several experimental signatures, based on data taken during the 2015 and part of 2016 runs are summarised.
\end{abstract}

55th International Winter Meeting on Nuclear Physics

23-27 January, 2017

Bormio, Italy

${ }^{*}$ Speaker, on behalf of the ATLAS and CMS Collaborations 


\section{Introduction}

After the discovery by ATLAS and CMS [1,2] of a particle compatible with the Standard Model (SM) Higgs boson at a mass of about $125 \mathrm{GeV}$ at the Large Hadron Collider (LHC) in 2012, the ATLAS and CMS experiments [3,4] have greatly intensified the search program for sign of physics beyond the SM (BSM). Exploiting the increase in energy and instantaneous luminosity of the LHC that restarted operations in 2015 at the new record energy of $13 \mathrm{TeV}$ and reached its design luminosity during the 2016 data campaign, both ATLAS and CMS have been able to engage a new set of measurements. The new conditions allowed to considerably improving the measurements of coupling strengths and structure, the spin/CP, mass, decay widths of the Higgs boson, and searching for new particles.

These proceedings summarise a small fraction of the preliminary results on Higgs boson physics and BSM searches based on Run 2 data collected by ATLAS and CMS at $\sqrt{s}=13 \mathrm{TeV}$ in 2015 and 2016.

\section{Higgs physics: H(125) Properties}

The ATLAS and CMS experiments combined their Higgs-boson mass measurements in fully reconstructed $\mathrm{H} \rightarrow \mathrm{ZZ} \rightarrow 4 \mathrm{l}$ and $\mathrm{H} \rightarrow \gamma \gamma$ channels to produce the Run 1 LHC average mass value of $\mathrm{m}_{H}=125.09 \pm 0.24 \mathrm{GeV}$ [5] . Measurements of the couplings to SM particles such as $\mathrm{W}$ and $\mathrm{Z}$ bosons, $\mathrm{t}, \mathrm{b}$ and $\tau$ fermions, as well as $\mu$ leptons from both experiments were also combined. The combination of the measurement of the couplings was based on the measured $\sigma \times \mathrm{BR}(\mathrm{H} \rightarrow \mathrm{XX})$ for all investigated Higgs boson decay modes, i.e. $\mathrm{H} \rightarrow \gamma \gamma, \mathrm{ZZ}, \mathrm{WW}, \mathrm{bb}, \tau \tau$ and $\mu \mu$ [6]. The combined signal yield relative to the SM expectation is $\mu=1.09 \pm 0.11$, compatible with the SM expectation within the experimental uncertainty. The combination of the two experiments leads to observed significances of the VBF production process and of the $\mathrm{H} \rightarrow \tau \tau$ decay mode at more than 5 standard deviations. The combined significance for the ttH process was expected to be $2.0 \sigma$, whereas an excess of $4.4 \sigma$ was measured. Precise measurements of the Higgs boson coupling strengths to SM vector bosons and fermions constitute a stringent probe of various BSM Higgs scenarios. Measurements of Higgs boson couplings in Run 1 so far remain consistent with their $\mathrm{SM}$ predictions and are found to follow the expected mass scaling. Multiple $\mathrm{J}^{C P}$ scenarios of the observed Higgs boson were probed during Run 1 and results exclude all considered non-SM spin hypotheses at more than $99 \%$ confidence level (CL) [7, 8].

Run-2 data is also used to make fiducial and differential cross-section measurements. In particular the Higgs boson decay to four leptons provides good sensitivity to the measure its properties despite the small branching ratio $(0.0125 \%)$ due to the low background contribution. Table 1 reports the fiducial cross sections measured by ATLAS and CMS [9, 10, 11, 12]. With only a fraction of the total data statistic collected in 2016 CMS obtains a relative precision on the fiducial measurement already matching the precision achieved in Run 1. Differential cross section measurements have been performed as well by both ATLAS and CMS, and Figure 1 shows one example from the CMS experiment [12].

Next important step in the H(125) physics program of ATLAS and CMS is to establish the ttH associated production process to probe directly the $\mathrm{ttH}$ vertex, and to observe the $\mathrm{H} \rightarrow$ bb decay 
Table 1: Fiducial cross section measurements for $\mathrm{H} \rightarrow \mathrm{ZZ} * \rightarrow 41$ and $\mathrm{H} \rightarrow \gamma \gamma[9,10,11,12]$

$\begin{array}{cccc}\sqrt{s}=13 \mathrm{TeV} & \text { Channel } & \text { Measurement }(\mathrm{fb}) & \text { SM prediction }(\mathrm{fb}) \\ \text { ATLAS }\left(13.3 \mathrm{fb}^{-1}\right. & \mathrm{H} \rightarrow \gamma \gamma & 43.2 \pm 14.9 \text { (stat) } \pm 4.9 \text { (syst) } & 62.7_{-4.4}^{+3.4}(\mathrm{~N} 3 \mathrm{LO}+\mathrm{XH}) \\ \text { CMS }\left(12.9 \mathrm{fb}^{-1}\right. & \mathrm{H} \rightarrow \gamma \gamma & 69_{-22}^{+16} \text { (stat) }{ }_{-6}^{+8} \text { (syst) } & 73.8 \pm 3.8 \\ \text { ATLAS }\left(14.8 \mathrm{fb}^{-1}\right. & \mathrm{H} \rightarrow \mathrm{ZZ} * & 4.54_{-0.90}^{+1.02} & 3.07_{-0.25}^{+0.21} \\ \text { CMS }\left(12.9 \mathrm{fb}^{-1}\right. & \mathrm{H} \rightarrow \mathrm{ZZ} * & 2.29_{-0.64}^{+0.74} \text { (stat) }{ }_{-0.23}^{+0.30} \text { (syst) } & 2.53 \pm 0.13\end{array}$

mode. These analyses are in progress and preliminary results with the data sample collected in 2015 and part of 2016 are shown in Figure 2 [13, 14]. More data and study are still needed to establish these processes experimentally

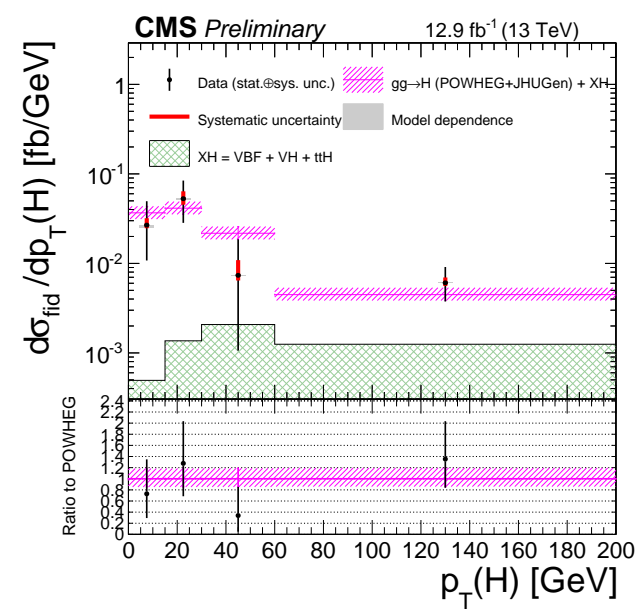

Figure 1: CMS result of the differential cross section measurement for $\mathrm{p}_{\mathrm{T}}(\mathrm{H})$. The acceptance and theoretical uncertainties in the differential bins are are calculated using POWHEG [10].

\section{Higgs physics: BSM Higgs Searches}

A multitude of BSM Higgs searches are carried out by CMS and ATLAS at $\sqrt{s}=13 \mathrm{TeV}$ in order to understand the structure of the Higgs sector: minimal as in the SM, or extended as predicted in several BSM models. The results of a few of such searches are illustrated below.

In the Minimal Supersymmetric Standard Model (MSSM) heavy Higgs bosons can have enhanced couplings to down-type fermions resulting in an increased production of the heavy Higgs boson that decays mainly to top quarks and tau leptons. A search for a neutral Higgs boson using the decay into two tau leptons has been presented by the CMS experiment [15]. The analysis uses $12.9 \mathrm{fb}^{1}$ of data collected in 2016, at a centre of mass energy of $13 \mathrm{TeV}$. The results are interpreted in the context of the minimal supersymmetric standard model. No excess above the expectation from the standard model is found and upper limits are set on the production cross sections times branching fraction for masses between 90 and $3200 \mathrm{GeV}$. Figure 3 shows the model dependent exclusion limits in the $m_{A}-\tan \beta$ plane, interpreted in the context of the $m_{\mathrm{h}}^{\bmod }{ }^{+}$scenario. A new ATLAS search sensitive to a broad range of BSM benchmark models (MSSM and general type-I,II, III $2 \mathrm{HDM}$ model) searched for anomalies in $t \bar{t}$ final states with additional heavy flavor jets using 

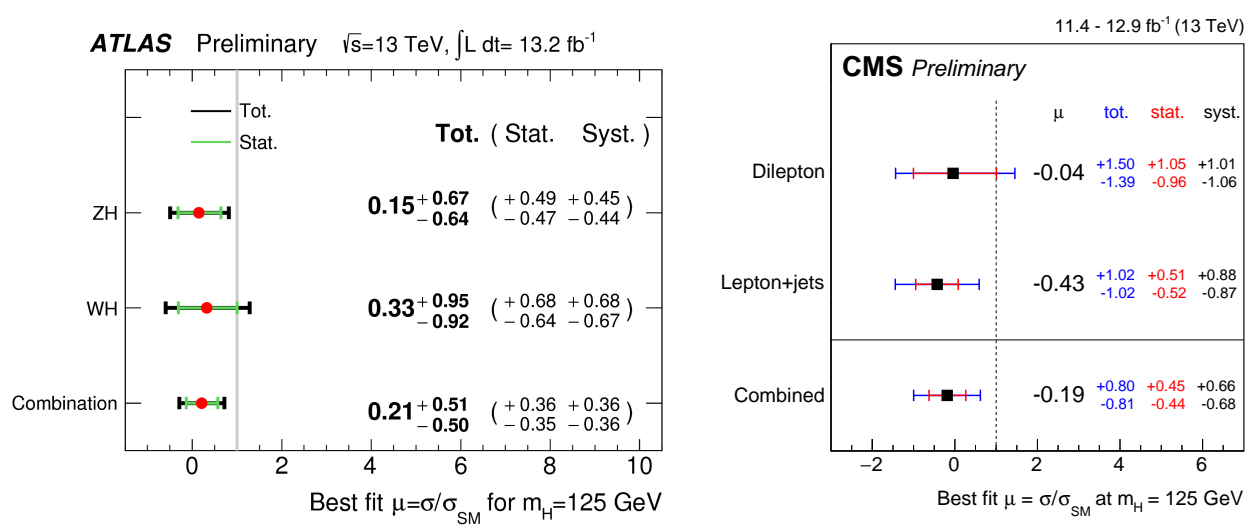

Figure 2: Measured signal strengths, normalised to the Standard Model expectations for ATLAS pp $\rightarrow$ $\mathrm{VH} \rightarrow$ Vbb decay [13] (left), and for CMS ttH production with $\mathrm{H} \rightarrow$ bb [14] (right).

$13.2 \mathrm{fb}^{-1}$ of collected data, finding good agreement with the SM expectations [16]. As example of cross-section upper limits at 95\% CL set for type-I and type-II 2HDM models as a function of the Higgs boson mass is shown in Figure 3.
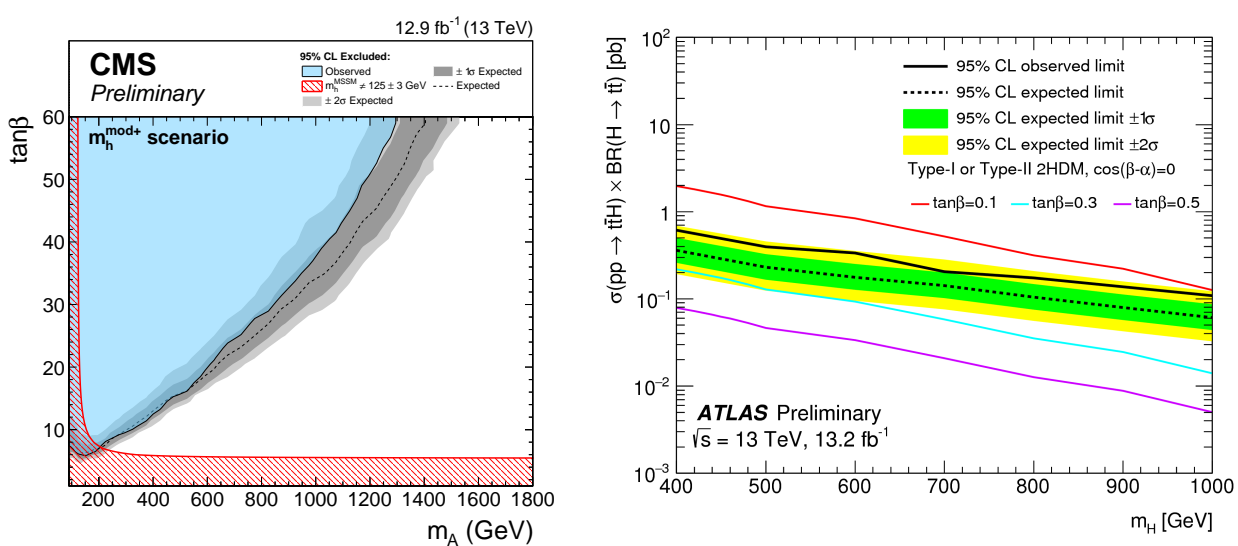

Figure 3: (Left) model dependent exclusion limits in the $m_{A}-\tan \beta$ plane, interpreted in the context of the $m_{\mathrm{h}}^{\text {mod }+}$ scenario from CMS neutral Higgs boson search described in [15]. (Right) ATLAS Observed (solid line) and expected (dashed line) 95\% CL upper limits on $\sigma(\mathrm{pp} \rightarrow \mathrm{ttH}) \times \mathrm{BR}(\mathrm{H} \rightarrow \mathrm{tt})$ as a function of the heavy Higgs boson mass $m_{\mathrm{H}}$, compared to the theoretical predictions assuming a Type-I or Type-II 2HDM. The surrounding shaded bands correspond to \pm 1 and \pm 2 standard deviations around the expected limit. The coloured thin lines show the theoretical predictions corresponding to different values of $\tan \beta$, assuming $\cos (\beta-\alpha)=0[16]$

In the SM the production cross section of Higgs boson pairs is order of magnitude below the experimental sensitivity, but di-Higgs production can be enhanced in BSM via modified coupling constant or new resonances participating to the process. Searches for heavy neutral scalar Higgs decaying into a pair of SM Higgs particles thus offer great potential in the search for new phenomena. New searches for a heavy neutral Higgs-bosons decaying to di-Higgs bosons are performed by both ATLAS and CMS at $\sqrt{s}=13 \mathrm{TeV}$ looking at Higgs decays in several final states [17, 18], ranging from bb $\gamma \gamma$ final states, that provide best discovery sensitivity at lower heavy Higgs masses, to de- 
cays in four b-quarks, that extends to high mass the explorable range tanks to the larger branching fraction and exploiting events with boosted topologies. Figure 4 show the upper limit for the pp $\rightarrow$ hh $\rightarrow$ bbbb ATLAS search, interpreted in the context of the bulk Randal-Sundrum model with $\mathrm{k} / \mathrm{MPl}=1$ at the $95 \%$ confidence level, based on $13.2 \mathrm{fb}^{-1}$ of data.

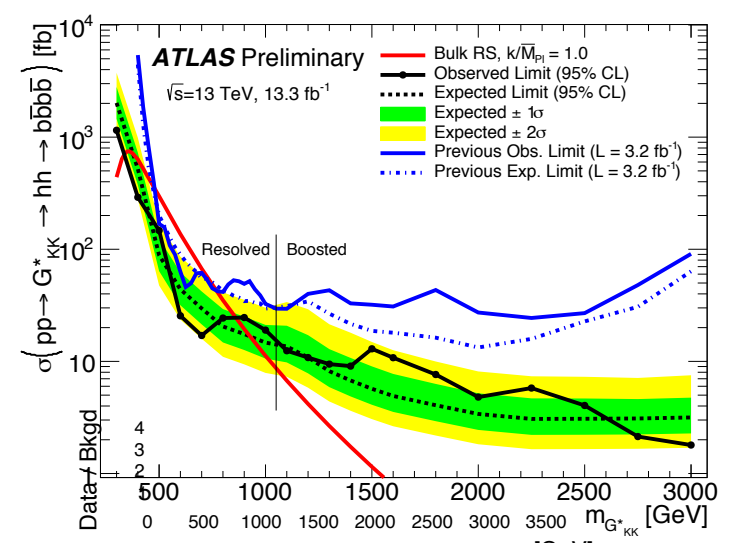

Figure 4: Expected and observed upper limit for $\mathrm{pp} \rightarrow \mathrm{G}^{*}{ }_{K K} \rightarrow \mathrm{hh} \rightarrow$ bbbb in the bulk RS model with $\mathrm{k} / \mathrm{MPl}=1$ at the $95 \%$ confidence level. The results of the resolved analysis are used up to a mass of 1000 $\mathrm{GeV}$ and those of the boosted analysis are used at higher mass where its expected sensitivity is higher. The red curves show the predicted cross sections as a function of resonance mass for the bulk RS model with $\mathrm{k} / \mathrm{MPl}=1$. The drop in cross section for masses below $\mathrm{m}_{\mathrm{G} *{ }_{\mathrm{K} K}}=350 \mathrm{GeV}$ is due to a sharp drop in the $\mathrm{BR}\left(\mathrm{G}^{*}{ }_{K K} \rightarrow \mathrm{hh}\right)[18]$.

\section{New Physics Searches: Supersymmetry}

A huge number of analyses have been performed or are near completion in ATLAS and CMS in the search for signals predicted by Supersymmetric models, two representative examples of such searches are discussed here. Searches can be generally divided in searches for strong and electroweak production mechanisms. In the case of strong production of squarks and gluinos, the cross-sections would be rather high, compared for example to production of similar-mass particles produced only via electroweak processes. For the strong production CMS has optimised the discovery sensitivity by categorising the events in several search regions based on the number of b-tagged jets, missing transverse momentum, hadronic transverse energy, and the invariant mass of opposite-sign, same-flavor dilepton pairs in the events. No significant excess above the standard model background expectation is observed and strong exclusion limits on the mass of the involved Supersymmetric particles are set. Figure 5 summarise the results of the CMS interpreted in the context of the stop-pair production, shown as exclusion region in the stop squark and LSP mass plane [19]. Searches for the direct electroweak production of supersymmetric charginos and neutralinos have been performed in final states with two light leptons of the same charge and with three or more leptons with large transverse momentum and no associated hadronic jets. Figure 5 (right) summarise the ATLAS results of electroweak production in terms of exclusion region in the electroweakino (charginos and heavy neutralino) VS LSP mass plane [20]. 

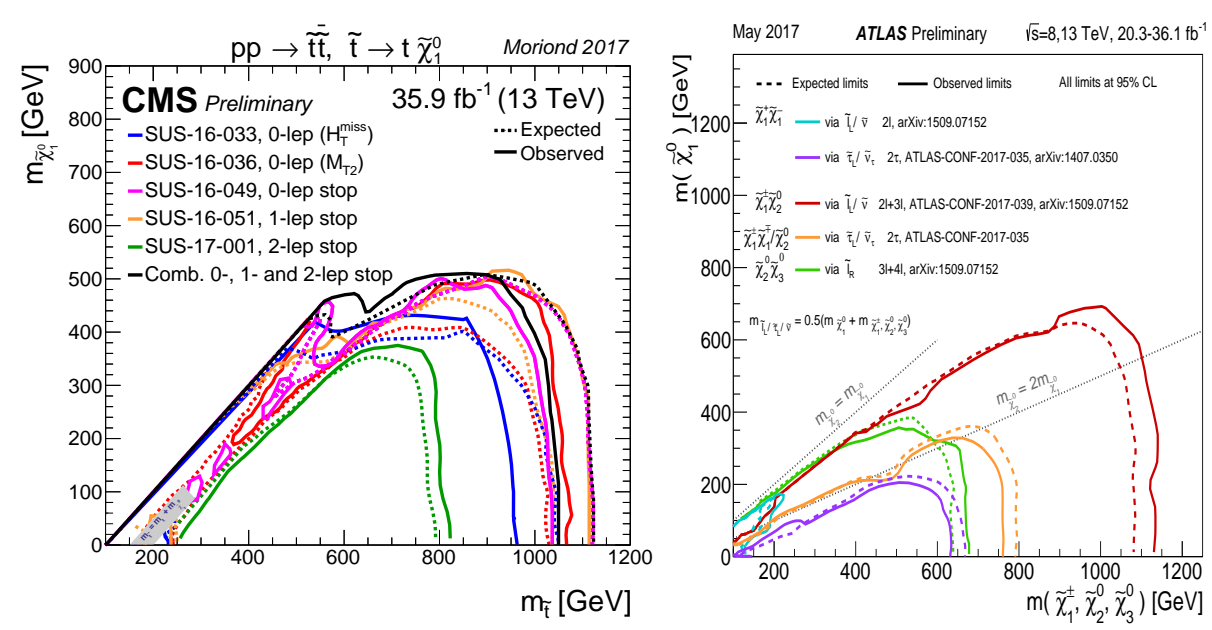

Figure 5: (Left) observed and expected exclusions in the stop squark and LSP mass plane for the CMS searches for strong production of Supersymmetric particles [19]. (Right) ATLAS 95\% CL exclusion limits on $\left.\chi_{1}^{+} \chi\right) 1^{-}, \chi_{1}^{ \pm} \chi_{2}^{0}$ and $\chi_{2}^{0} \chi_{3}^{0}$ production with $l$-mediate decays, as a function of the $\chi_{1}^{ \pm}, \chi_{2}^{0}$ and $\chi_{3}^{0}$ masses. The production cross-section is for pure wino $\left.\left(\chi_{1}^{+} \chi\right) 1^{-}, \chi_{1}^{ \pm} \chi_{2}^{0}\right)$ and pure higgsino $\left(\chi_{2}^{0} \chi_{3}^{0}\right)$ [20].

\section{New Physics Searches: Dijet Resonances}

The search for new physics in dijet final states is of primary importance in LHC. In fact In the SM dijet processes are described well by Quantum Chromodynamics. Any departure from SM expectations implies the existence of new physics. ATLAS and CMS have searched for new physics in dijet final states in both high mass and low mass regimes [21, 22]. The search for low mass dijets is limited by the requirements of the trigger designed to keep the rate of backgrounds events at an affordable level. One of the ways of avoiding this problem is to require an Initial State Radiation (ISR) jet or photon. Another approach is based on performing a trigger-level analysis, saving only a sub-set of the physics objects immediately after the High Level Trigger selection. In this way it is possible to sustain the high event rate typical of the dijet triggers. Figure 6 shows the $\mathrm{m}_{\mathrm{jj}}$ distribution for events with a photon of transverse momentum $\mathrm{p}_{\mathrm{jT}}>150 \mathrm{GeV}$ and two jets with $\mathrm{p}_{\mathrm{jT}}>25 \mathrm{GeV}$ reconstructed by the ATLAS experiment [21]. The vertical lines represent the interval identified to contain the highest discrepancy between the data and the predicted background.

The search for high mass dijets is carried out by looking for an excess of data over a steeply falling dijet background as done in the low mass searches. Figure 7 shows the results of the CMS analysis [22] based on $15.5 \mathrm{fb}^{-1}$ of integrated luminosity collected at $\sqrt{s}=13 \mathrm{TeV}$, and the exclusion limits set on generic quark-quark, quark-gluon and gluon-gluon resonances interpreted in the context of several NP models.

\section{New Physics Searches: Dark Matter}

The ATLAS and CMS program of searches for Dark Matter (DM) is competitive and complementary to the direct detection experiments via DM-nucleon scattering and to the indirect detection experiments via DM annihilation. Although with a certain level of model dependence, ATLAS and CMS have probed a wide range of DM-SM interactions employing different search strategies. One 


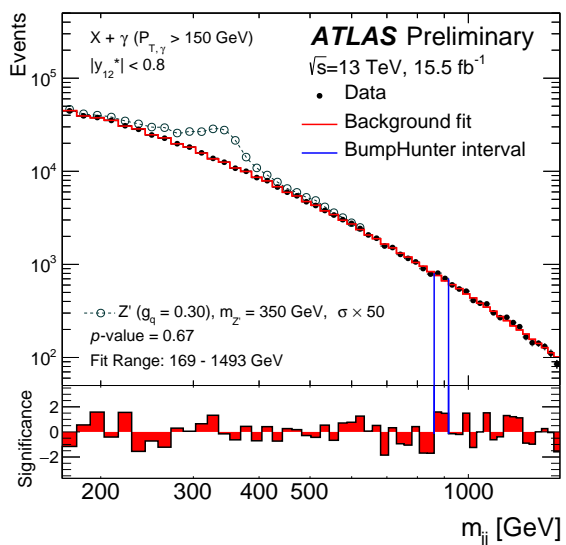

Figure 6: The $\mathrm{m}_{\mathrm{jj}}$ distribution for events with a photon of transverse momentum $\mathrm{p}_{\mathrm{jT}}>150 \mathrm{GeV}$ and two jets with $\mathrm{p}_{\mathrm{j} T}>25 \mathrm{GeV}$ reconstructed by the ATLAS experiment [21].
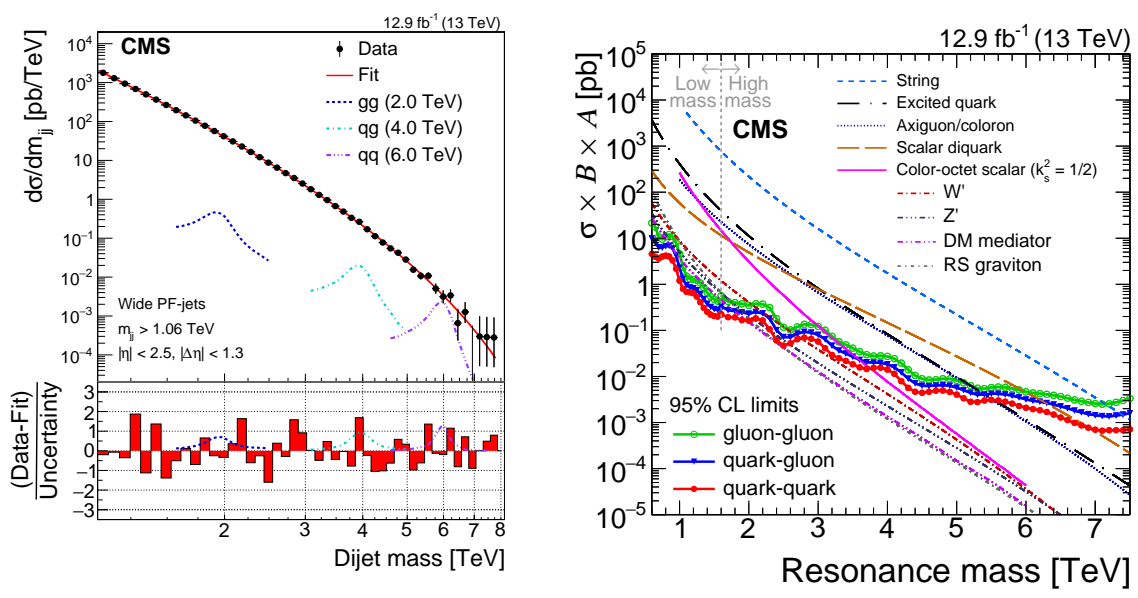

Figure 7: (Left) the dijet mass spectrum compared to a fitted parametrisation of the background in the high mass regime from CMS high-mass dijet search. (Right) summary of the exclusion limits in the high mass dijet regime from CMS [22].

typical approach is the search for events in which DM is pair-produced in association with another object, for example and ISR jet or photon. As the DM is invisible in the detector the signature of the DM in these events is a significant imbalance in the transverse momentum $E_{\mathrm{T}}^{\text {miss }}$ of the event. This class of searches is typically referred to as Mono-X searches. In a complementary approach indirect constraint on the DM is obtained by searching for mediators which could connect the SM to the DM, exploiting the fact that any SM process that can form the dark mediator can also decay back to the input SM particles. A typical example is the search for dijet resonances described in the previous section where it is assumed that the mediator couples to light quarks or gluons, and an observed resonance could be interpreted as the production of a new dark mediator.

Concerning the Mono-X searches, the final state with the highest SM cross-section is the $E_{\mathrm{T}}^{\text {miss }}$ + jet analysis, where the large rate for high transverse momentum ISR jets is exploited to obtain the strongest vector and axial-vector mediator limits. Primary background for this search is the 
irreducible $Z \rightarrow v v+$ jets process. A not negligible discovery sensitivity comes also from the $E_{\mathrm{T}}^{\text {miss }}+$ photon search that has smaller cross-section but a cleaner final-state. The most sensitive search channel for scalar mediators with mass below $100 \mathrm{GeV}$ is the mono-t $\bar{t}$ channel. In this case the dominant source of background is the SM $t \bar{t}$ production with with semileptonic $t \bar{t}$ events in the hadronic mono-t $t \bar{t}$ search and fully leptonic $t \bar{t}$ events in the semileptonic mono-t $t \bar{t}$ search. Sensitivity for this searches has been recently improved by CMS using novel resolved-hadronic-top-tagging techniques and by categorising hadronic channel events by expected signal purity [23].
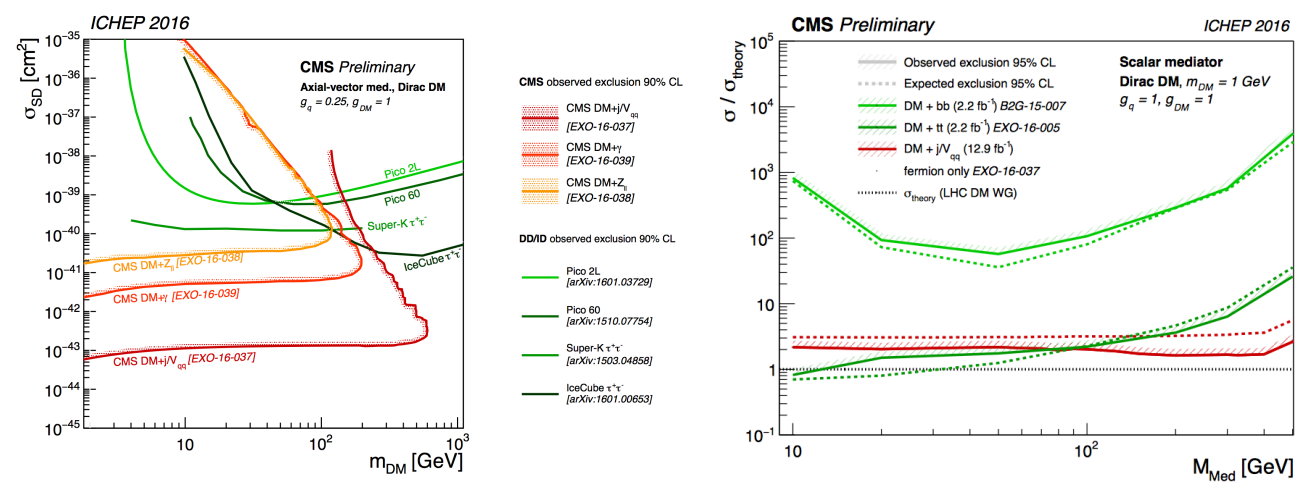

Figure 8: (Left) a comparison of the CMS results of the mono-jet, mono- $\gamma$ and mono-Z searches displayed as 90\% CL exclusions in the DM mass - spin-dependent DM-nucleon cross section, interpreted in the in the context of a simplified model with an Axial Vector mediator, Dirac DM and couplings $q_{g}=0.25$ and $g_{\mathrm{DM}}$ $=1$. The exclusion contour is compared with limits from the PICO experiments, the IceCube limit for the $t \bar{t}$ annihilation channel and the Super-Kamiokande limit for the $b \bar{b}$ annihilation channel. (Right) the 95\% CL observed (full-line) and expected (dashed-line) exclusion limits for the scalar model as a function of mediator mass for different $E_{\mathrm{T}}^{\text {miss }}$ based DM searches from CMS [24, 23].

In Figure 8 (left) the CMS results of the mono-jet, mono- $\gamma$ and mono-Z searches are displayed as $90 \%$ CL exclusions in the DM mass - spin-dependent DM-nucleon cross section, interpreted in the in the context of simplified model with an Axial Vector mediator, Dirac DM and couplings $q_{\mathrm{g}}=0.25$ and $g_{\mathrm{DM}}=1$. [24]. It should be noted that the CMS limits do not include a constraint on the relic density and also the absolute exclusion of the different CMS searches as well as their relative importance will strongly depend on the chosen coupling and model scenario. Therefore, the shown CMS exclusion regions in this plot are not applicable to other choices of coupling values or models. Figure 8 (right) shows the exclusion limits for the scalar model as a function of the mediator mass from various CMS mono-X searches, assuming $g_{\mathrm{q}}=1$ and $g_{\mathrm{DM}}=1$.For smaller mediator masses, the mono-t $\bar{t}$ search already has better sensitivity than the mono-jet search even with the 2015 dataset [23].

The ATLAS bounds in the coupling-mediator mass plane obtained from the dijet searches using 2015 and 2016 data are presented in Figure 9. In the left figure are reported the regions in the dark matter mass-mediator mass plane excluded at $95 \%$ CL by a selection of ATLAS dark matter searches [25, 26, 27, 27], for one possible interaction between the Standard Model and dark matter, the leptophobic axial-vector mediator as described in [28]. The exclusions are computed for a dark matter coupling $g_{\mathrm{DM}}=1.0$, a quark coupling $g_{\mathrm{q}}=0.25$ universal to all flavors. The lepton coupling $g_{1}$ in this model is set to zero. This choice for the couplings corresponds to the "A1" scenario 
in [28].
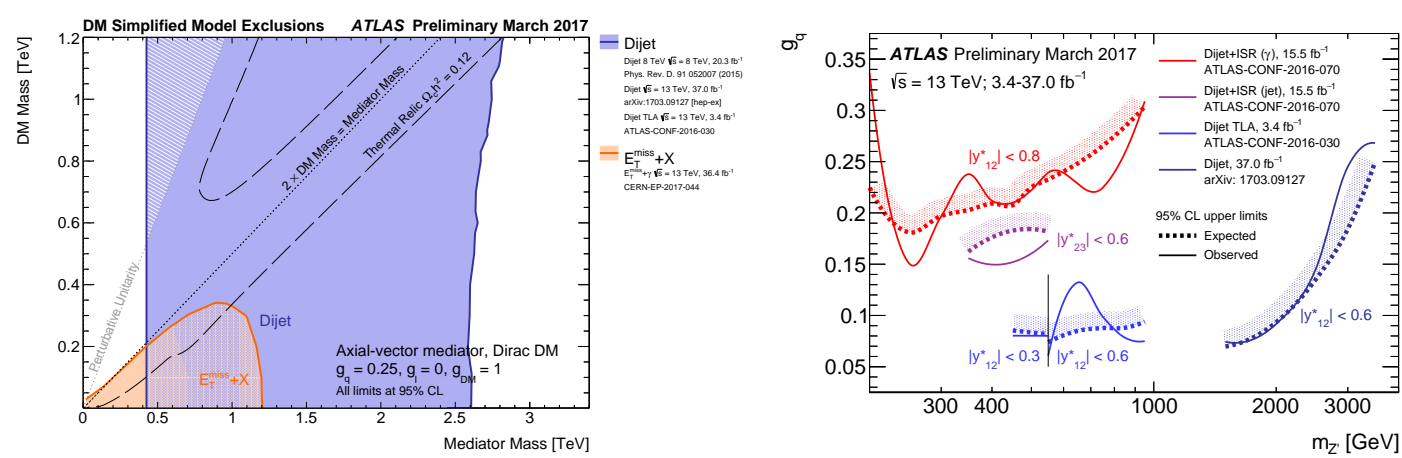

Figure 9: (Left) regions in a dark matter mass-mediator mass plane excluded at 95\% CL by a selection of ATLAS dark matter searches, for one possible interaction between the Standard Model and dark matter, the leptophobic axial-vector mediator. Dashed curves labeled "thermal relic" indicate combinations of dark matter and mediator mass that are consistent with a dark matter density of $\Omega_{c}=0.12 \mathrm{~h}^{2}$ and a standard thermal history, as computed in MadDM. Between the two curves, annihilation processes described by the simplified model deplete $\Omega_{c}$ below $0.12 \mathrm{~h}^{2}$. A dotted curve indicates the kinematic threshold where the mediator can decay on-shell into dark matter. Excluded regions that are in tension with the perturbative unitary considerations of [31] are indicated by shading in the upper left. The exclusion regions, relic density contours, and unitarity curve are not applicable to other choices of coupling values or model. (Right) Summary plot of ATLAS bounds in the coupling-mediator mass plane from dijet searches using 2015 and 2016 data. The 95\% CL upper limits are obtained from three ATLAS searches using 2015 and 2016 data on coupling $g_{\mathrm{q}}$ as a function of the resonance mass $m_{Z^{\prime}}$ for the leptophobic $Z^{\prime}$ model. The expected limits from each search are indicated by dotted curves. [25, 26, 27, 29].

In Figure 9 right, the 95\% CL upper limits obtained from three ATLAS searches [26, 27, 29] using 2015 and 2016 data on coupling $g_{\mathrm{q}}$ as a function of the resonance mass $\mathrm{m}_{\mathrm{Z}^{\prime}}$ for the leptophobic Z' model described in [30] are shown. The darker blue curves show the results from a "high-mass" search using the traditional triggering strategy for dijet resonances, employing the lowest-transverse-momentum-threshold single-jet trigger without prescales. The red curves show the results from a search triggering on events containing a photon, such as those radiated from the initial state partons that would produce the resonance, while the purple curves show the results from a search for the case when the initial state radiation is instead a jet, recorded by the lowesttransverse-momentum-threshold single-jet trigger without prescales. The lighter blue curves show the results from a Trigger-object Level Analysis of events triggered with much lower jet transverse momenta than the high-mass result. The selections in the rapidity difference $\left(y *=1 / 2\left(y_{i}-y_{j}\right)\right)$ of the two jets used to reconstruct the invariant mass are also shown, for the three searches. Coupling values above the solid curves are excluded, as long as the signals are narrow enough to be detected using these searches (10\% signal width / mass for dijet+ISR and TLA, 15\% for high-mass dijets, approximately corresponding to $g_{\mathrm{q}}<0.5$ and $g_{\mathrm{q}}<0.6$, respectively).

\section{New Physics Searches: Long-lived Particles}

Almost all the conceivable extensions of the Standard Model predict the existence of new, long-lived particles (LLP), which would appear with very distinctive experimental signatures that 

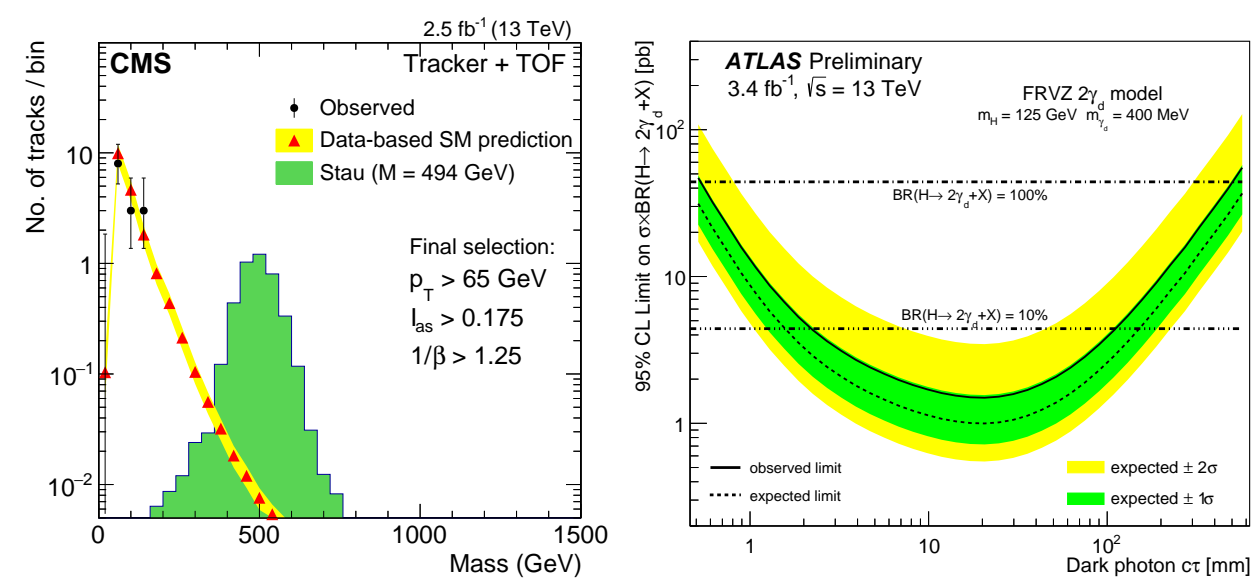

Figure 10: (Left) CMS observed and predicted mass spectra for candidates HSCPs from mGMSB model with stau slepton as the next-to-lightest supersymmetric particle [32]. The expected distributions for the benchmark signal is shown as histogram. (Right) ATLAS 95\% upper limits on the $\sigma \times \mathrm{BR}$ for the FRVZ 125 $\mathrm{GeV}$ Higgs $\rightarrow 2 \gamma_{d}+\mathrm{X}$ benchmark modes as a function of the dark-photon lifetime (c $\tau$ ). The horizontal lines correspond to $\sigma \times \mathrm{BR}$ for two values of the BR of the Higgs boson decay to dark photons [33].

offers a means for new physics to escape the strict limits placed by prompt searches and at the same time a great potential for discovery. Scenarios in which these new particles could arise include supersymmetric scenarios such as split-SUSY or SUSY with very weak R-parity violation, Hidden Valley models or dark sector models, and Z' models that include long-lived neutralinos. ATLAS and CMS are in an extensive program of searches for LLP decays. Here two representative examples of these searches are presented. Figure 10 (left) shows the observed and predicted spectra of candidates of Heavy stable charged particles (HSCP), in a search for long-lived particles which have a sufficiently long lifetime to leave the CMS detector before decaying. These particles can have a speed significantly less than thee speed of light, or a charge Q not equal to the electron charge, or both. The analysis relies on using the time-of-flight (TOF) of the particles to the muon system and/or the rate of specific ionisation $(\mathrm{dE} / \mathrm{dx})$ measured in the tracker for the identification of HSCPs [32]. No significant deviations from the expectation of the SM has been observed by CMS in $2.5 \mathrm{fb}^{-1}$ of data at $13 \mathrm{TeV}$, and upper limits are set on the cross sections for production of long-lived gluinos, top squarks, tau sleptons, and leptonlike long-lived fermions. These upper limits are equivalently expressed as lower limits on the masses of new states; the limits for gluinos, ranging up to $1610 \mathrm{GeV}$, are the most stringent to date.

Figure 10 (right) presents the result of a search for long-lived neutral particles decaying into collimated jets of light leptons and mesons, so-called "lepton-jets", using a sample of $3.4 \mathrm{fb}^{\text {?1 }}$ of proton-proton collisions data at a centre-of-mass energy of $13 \mathrm{TeV}$ collected during 2015 with the ATLAS detector at the LHC [33]. No deviations from Standard Model expectations are observed. Limits on models predicting Higgs boson decays to neutral long-lived particles (dark photons $\gamma_{d}$ ), which in turn produce lepton-jets, are derived as a function of the particle proper decay length, $c \tau$. Assuming the Standard Model gluon-fusion production cross section for a $125 \mathrm{GeV}$ Higgs boson, its branching ratio to dark photons is found to be below 10, at $95 \% \mathrm{CL}$, for dark-photon lifetimes 
in the range $2.2 \leq \mathrm{c} \tau \leq 111.3 \mathrm{~mm}$ in a Higgs $\rightarrow 2 \gamma_{d}+\mathrm{X}$ benchmark model and in the range 3.8 $\leq \mathrm{c} \tau \leq 163 \mathrm{~mm}$ in a Higgs $\rightarrow 4 \gamma_{d}+\mathrm{X}$ model. These limits improve upon the results of a similar search previously performed during Run 1 of the LHC, due to enhancements in the trigger and reconstruction of highly-collimated muons, and are the most stringent to date in the explored mass and lifetime range of the dark-photon.

\section{References}

[1] ATLAS Collaboration, Phys. Lett. B 716, 1, 2012.

[2] CMS Collaboration, Phys. Lett. B 716, 30, 2012.

[3] ATLAS Collaboration, JINST 3 S08003 (2008).

[4] CMS Collaboration, JINST 3 S08004 (2008).

[5] ATLAS and CMS Collaborations, Phys. Rev. Lett. 114, 191803 (2015).

[6] ATLAS and CMS Collaborations, ATLAS-CONF-2015-044/CMS-PAS-HIG-15-002 (2015).

[7] ATLAS Collaboration, Eur. Phys. J. C75 (2015) 476.

[8] CMS Collaboration, Phys. Rev. D 92 (2015) 012004.

[9] ATLAS Collaboration, ATLAS-CONF-2016-067 (2016).

[10] CMS Collaboration, CMS-PAS-HIG-16-020 (2016).

[11] ATLAS Collaboration, ATLAS-CONF-2016-079 (2016).

[12] CMS Collaboration, CMS-PAS-HIG-16-033 (2016).

[13] ATLAS Collaboration, ATLAS-CONF-2016-091 (2016).

[14] CMS COllaboration, CMS-PAS-HIG-16-038 (2016).

[15] CMS Collaboration, CMS-PAS-HIG-16-037 (2016).

[16] ATLAS Collaboration, ATLAS-CONF-2016-104 (2016).

[17] CMS Collaboration, CMS-PAS-HIG-16-011, CMS-PAS-HIG-16-029, CMS-PAS-HIG-16-002, CMS-PAS-HIG-16-032 (2016).

[18] ATLAS Collaboration, ATLAS-CONF-2016-049 (2016).

[19] CMS Collaboration, CMS-PAS-SUS-16-033, CMS-PAS-SUS-16-036, CMS-PAS-SUS-16-049, CMS-PAS-SUS-16-051 (2016), and CMS-PAS-SUS-17-001 (2017).

[20] ATLAS Collaboration, Phys. Rev. D 93, 052002 (2016), JHEP10(2014)096, Phys. Rev. D 93, 052002 (2016), ATLAS-CONF-2017-035 (2016), Phys. Rev. D 93, 052002 (2016).

[21] ATLAS Collaboration, ATLAS-CONF-2016-070 (2016), ATLAS-CONF-2016-070 (2016).

[22] CMS Collaboration, arXiv:1611.03568 [hep-ex].

[23] CMS Collaboration, CMS-PAS-EXO-16-005 (2016).

[24] CMS Collaboration, CMS-PAS-EXO-16-037 (2016), CMS-PAS-EXO-16-038 (2016), CMS-PAS-EXO-16-039 (2016).

[25] ATLAS Collaboration, Phys.Rev.D. 91052007 (2015) 
[26] ATLAS Collaboration, arXiv:1703.09127 [hep-ex].

[27] ATLAS Collaboration, ATLAS-CONF-2016-030 (2016).

[28] A. Albert et al., arXiv:1703.05703 [hep-ex].

[29] ATLAS Collaboration, ATLAS-CONF-2016-070 (2016).

[30] D. Abercombie et al., arXiv:1507.00966 [hep-ex].

[31] F. Kahlhoefer, K. Schmidt-Hoberg, T. Schwetz, S. Vogl, JHEP 1602 (2016) 016.

[32] CMS Collaboration, Phys. Rev. D 94 (2016) 112004.

[33] ATLAS Collaboration, ATLAS-CONF-2016-042 (2016). 